\title{
Hepatic Hydrothorax: The Conundrum and the Oxymoron
}

\author{
Sachin Kumar ${ }^{1}$ Yashwant Patidar $^{2} \quad$ Rakhi Maiwall $^{3}$ \\ 1 Department of Pulmonary Medicine, Institute of Liver and Biliary \\ Sciences, New Delhi, India \\ 2 Department of Radiodiagnosis, Institute of Liver and Biliary Sciences, \\ New Delhi, India \\ ${ }^{3}$ Department of Hepatology, Institute of Liver and Biliary Sciences, \\ New Delhi, India

\begin{abstract}
Address for correspondence Sachin Kumar, MD, DM, FCCP, Department of Pulmonary Medicine, Institute of Liver and Biliary Sciences, Vasant Kunj, Delhi 110070, New Delhi, India (e-mail: sachin.drk@gmail.com).
\end{abstract} \\ J Clin Interv Radiol ISVIR 2017;1:46-48.
}

\begin{abstract}
Hepatic hydrothorax $(\mathrm{HH})$ is an infrequent but a well-known complication of portal hypertension. Medical management of this condition often fails, and there are no large randomized-controlled trials establishing the best treatment strategies. $\mathrm{HH}$ thus represents a formidable entity in the management of end-stage liver disease, and the only definitive treatment is liver transplantation. Despite documented ominous outcome, tube thoracostomy (TT) is still a widely practiced approach to $\mathrm{HH}$ mostly by the

Keywords

- hepatic hydrothorax

- chest tube removal

- fistula

- cirrhosis unaware primary care physician. This communication reports the occurrence of pleurosubcutaneous fistula as a presenting complication of TT in a patient with $\mathrm{HH}$. TT should thus be avoided at all costs in this subset of patients because it is fraught with multiple complications. This report reinforces the importance of clinical education and awareness of these complications and outcomes of TT in a cirrhotic patient with $\mathrm{HH}$.
\end{abstract}

\section{Introduction}

Hepatic hydrothorax $(\mathrm{HH})$ is defined as a transudative pleural effusion, usually more than $500 \mathrm{~mL}$, in a cirrhotic patient without an underlying cardiopulmonary or pleural disease. ${ }^{1}$ The estimated prevalence of $\mathrm{HH}$ is approximately 4 to $6 \%$ in cirrhotics. The only definitive treatment of $\mathrm{HH}$ is liver transplantation. Other management strategies include sodium restriction, diuretics, thoracentesis, transjugular intrahepatic portosystemic shunt (TIPS), pleurodesis, and video-assisted thoracic surgery. However, their role is limited to alleviate symptoms and prevent complications of $\mathrm{HH}$ in patients awaiting transplant, or to palliate those who are not transplant candidates. $^{2}$

Tube thoracostomy (TT) in patients with HH should be avoided at all costs because it is fraught with multiple complications such as massive fluid loss with secondary acute kidney injury, electrolyte and protein depletion, pneumothorax, hemothorax, empyema, and secondary infection that can eventually be fatal. ${ }^{2,3}$ Nevertheless, this procedure is still performed with the temptation to relieve breathlessness and to avoid repeated therapeutic thoracentesis in a patient with refractory $\mathrm{HH}$.

This report describes a hitherto unreported complication of TT for HH presenting as a reducible thoracic wall lump.

\section{Case Report}

A 41-year-old man with a background of cryptogenic cirrhosis presented with a 1-month history of progressive breathlessness. He was initially evaluated at a primary care center, where initial chest radiography showed a massive right-sided pleural effusion with contralateral mediastinal shift. Pleural fluid aspiration revealed straw-colored transudative fluid. However, a 24-Fr chest tube was inserted in the midaxillary line by the treating unit to alleviate the symptomatic patient. One and half liter of fluid was drained and he was referred to our hospital after chest tube removal for further management.

At the time of presentation, the patient was slightly breathless. His physical examination was remarkable for the presence of a lump over the right lateral chest wall. The lump had soft fluidlike fluctuant consistency and its
DOI https://doi.org/ 10.1055/s-0037-1603566. ISSN $0000-0000$
Copyright (c) 2017 by Indian Society of Vascular and Interventional Radiology
License terms

(요 (1) $\Theta \circledast$ 
dimensions were variable according to inspiration and expiration phases of respiration.

Chest X-ray showed gross right pleural effusion, and chest ultrasound revealed communication between the pleural cavity and chest wall characteristic of pleurosubcutaneous fistula (PSF) in the background of the cirrhotic liver (-Fig. 1). Noncontrast chest computed tomography also demonstrated gross right pleural effusion, and fluid collection was seen beyond the rib margin deep to the right lateral chest wall muscle.

The final diagnosis of a PSF secondary to TT for HH was made. The patient was put on a sodium-restricted diet, and maximal tolerable doses of diuretics were started. He eventually underwent TIPS for his refractory hydrothorax. Gradually, the size of lump decreased and pleural effusion also decreased considerably with good symptom control. Currently, he is listed for liver transplantation at our center.

\section{Discussion}

Pleurosubcutaneous fistula is defined as a pathological communication between the pleural space and the subcutaneous tissues. It can occur as a complication of an infectious process, neoplasm, foreign body aspiration, or iatrogenic procedures. ${ }^{4}$
Although, isolated case reports of PSF secondary to TT have been reported with malignant and tubercular pleural effusion. $^{4,5}$ it has not been described in patients with pleural effusion of other benign etiologies as $\mathrm{HH}$.

$\mathrm{HH}$ is an infrequent but a well-known complication of portal hypertension in patients with end-stage liver disease. Most of the studies have suggested the pathological transdiaphragmatic passage of ascitic fluid from the peritoneal to the pleural cavity through numerous diaphragmatic defects to be the predominant mechanism in the formation of $\mathrm{HH}^{6,7}$ Medical management often fails, and there is no large randomized-controlled trials establishing the best treatment options and other management strategies. HH thus represents a formidable entity in the management of end-stage liver disease.

Chest tubes are sometimes used as treatment for $\mathrm{HH}$, although patients with cirrhosis may have increased morbidity with TT, with difficulty in removing the tube without correction of the underlying liver disease or portal hypertension. ${ }^{3}$

Apart from single-case reports, the existing literature on the outcomes of cirrhotic patients who had chest tubes placed is limited to two large series. ${ }^{2,3}$ All except 1 patient in the first series of 17 patients suffered a complication related to TT for $\mathrm{HH}$, and overall 3-month mortality was $35 \%$. A study by Liu et

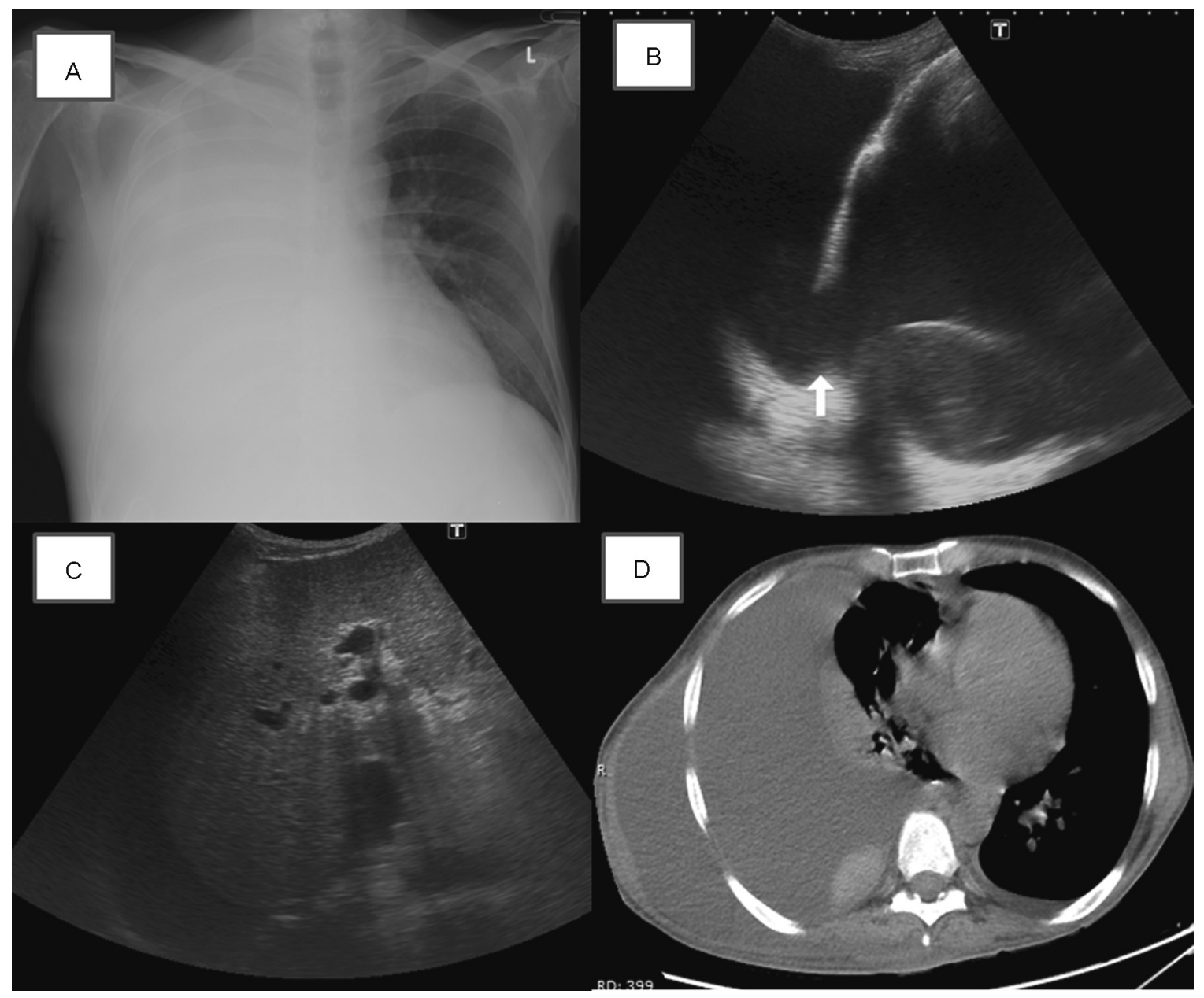

Fig. 1 (A) Chest X-ray showed gross right pleural effusion with soft tissue opacity along the right lateral chest wall. Chest ultrasound revealed communication between the pleural cavity and chest wall characteristic of pleurosubcutaneous fistula (B) and underlying cirrhotic liver (C). Axial image from the noncontrast chest computed tomography also demonstrated gross right pleural effusion, and fluid collection was seen beyond the rib margin deep to right lateral chest wall muscle (D). 
Table 1 Outcome of TT in cirrhotic

\begin{tabular}{|c|c|c|c|c|c|c|}
\hline Study & $N$ & $\mathrm{HH}$ & TIPS & Complications & Incidence & Mortality \\
\hline Orman and Lok $^{2}$ & 17 & $17(100)$ & $7(41.2)$ & $\begin{array}{l}\text { AKI } \\
\text { Hemodialysis (\% of AKI) } \\
\text { Pneumothorax } \\
\text { Empyema } \\
\text { Encephalopathy }\end{array}$ & $\begin{array}{l}11(64.7) \\
1(9.1) \\
7(41.2) \\
5(29.4) \\
3(17.6)\end{array}$ & $6(35.3)$ \\
\hline Liu et $\mathrm{al}^{3}$ & 59 & $24(42)$ & $1(2)$ & $\begin{array}{l}\text { (in children } B \text { and } C, N=56 \text { ) } \\
\text { Renal failure } \\
\text { Electrolyte imbalances } \\
\text { Infection }\end{array}$ & $\begin{array}{l}30(54) \\
32(57) \\
27(48)\end{array}$ & $15(27)$ after $1-39 \mathrm{~d}$ \\
\hline
\end{tabular}

Abbreviations: AKI, acute kidney injury; HH, hepatic hydrothorax; TIPS, transjugular intrahepatic portosystemic shunt; TT, tube thoracostomy. Note: Values are expressed as number (\%).

al reported an $80 \%$ overall complication rate and $27 \%$ mortality $^{2,3}$ ( - Table 1). Taken together, the literature suggests that chest tubes should not be placed to relieve HH because of the significant morbidity and mortality associated with the procedure.

In our patient, cirrhosis itself being a malnourished state compounded by impaired wound healing might be the predisposing factor for PSF. ${ }^{8,9}$ Significant residual pleural effusion after removal of the chest tube could also have contributed to the formation of PSF.

Although rare, this communications highlights the occurrence of hitherto unreported complication of TT in a cirrhotic patient with $\mathrm{HH}$, which might manifest itself only when the tube is removed, much to the dismay of the treating physician and the patient. The fact that physicians continue to perform drainage of $\mathrm{HH}$ reinforces the importance of physician education and awareness of these complications and outcomes despite documented poor outcome. ${ }^{2}$

In conclusion, we describe a case of PSF after TT in a cirrhotic patient with $\mathrm{HH}$. In a cirrhotic patient, drainage should be avoided as far as possible.

\section{Learning Pearls}

- HH is an infrequent but a well-known complication of portal hypertension in patients with end-stage liver disease.

- Management strategies include sodium restriction, diuretics, thoracentesis, TIPS, pleurodesis, and video-assisted thoracic surgery in selected patients. However, medical management of this condition often fails. HH thus represents a formidable entity in the management of end-stage liver disease, and liver transplantation remains the ultimate definitive management paradigm.

- Chest tube insertions should be avoided at all costs because they are fraught with multiple complications such as massive fluid loss with secondary acute kidney injury, electrolyte and protein depletion, hepatorenal syndrome, and secondary infection that can eventually be fatal.
- Despite documented poor outcome, primary care physicians still continue to perform tube drainage of $\mathrm{HH}$. This communication reinforces the importance of physician education and awareness of these complications and outcomes.

\section{Conflict of Interest}

The authors declare that they have no conflicts of interest in relation to this article.

\section{Author Contribution}

Sachin Kumar and Rakhi Maiwall were involved in patient management, preparation of manuscript, and literature search. Yashwant Patidar was involved in diagnostic radiology, manuscript preparation, and literature search.

\section{References}

1 Krok KL, Cárdenas A. Hepatic hydrothorax. Semin Respir Crit Care Med 2012;33(1):3-10

2 Orman ES, Lok AS. Outcomes of patients with chest tube insertion for hepatic hydrothorax. Hepatol Int 2009;3(4):582-586

3 Liu LU, Haddadin HA, Bodian CA, et al. Outcome analysis of cirrhotic patients undergoing chest tube placement. Chest 2004; 126(1):142-148

4 Lin MT, Shih JY, Lee YC, Yang PC. Pleurocutaneous fistula after tube thoracostomy: sonographic findings. J Clin Ultrasound 2008; 36(8):523-525

5 Navani N, Punwani S, Humphries PD, Booth HL. Pleuro-cutaneous fistula complicating chest drain insertion for tuberculous effusion. QJM 2010;103(10):799-800

6 Chen A, Ho YS, Tu YC, Tang HS, Cheng TC. Diaphragmatic defect as a cause of massive hydrothorax in cirrhosis of liver. J Clin Gastroenterol 1988;10(6):663-666

7 Kumar S, Kumar R. Hepatic hydrothorax: the shower within. J Bronchology Interv Pulmonol 2014;21(1):88-89

8 Nakamura R, Watanabe M, Sugimura Y, et al. Changes in wound healing factors in liver cirrhosis after esophageal transection for esophageal varices. Hepatogastroenterology 1995;42(1):43-46

9 Tsiaousi ET, Hatzitolios AI, Trygonis SK, Savopoulos CG. Malnutrition in end stage liver disease: recommendations and nutritional support. J Gastroenterol Hepatol 2008;23(4):527-533 